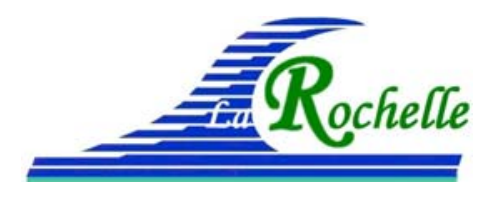

XV'̀mes Journées Nationales Génie Côtier - Génie Civil

La Rochelle, 29 au 31 mai 2018

DOI:10.5150/jngcgc.2018.052

(c) Editions Paralia CFL

disponible en ligne - http://www.paralia.fr - available online

\title{
Ouvrage de protection pour le port du Grand Large de Dunkerque à paroi perforée et partiellement transmissible
}

\author{
Julie LEBUNETEL ${ }^{1}$, Anne HONNEUR ${ }^{1}$, Andrès JEREZ ${ }^{1}$
}

1. INGEROP Conseil et Ingénierie, Agence de Rennes, 12 rue du Pâtis Tatelin, CS 50891, 35708 Rennes cedex 7, France.

julie.lebunetel@ingerop.com

\section{Résumé :}

L'extension du port de plaisance du Grand Large de Dunkerque nécessite la réalisation d'un ouvrage de protection spécifique. La solution d'ouvrage fixe est retenue dans un site contraint par un marnage important et des houles et clapots variés $(\mathrm{Tp}<7 \mathrm{~s})$. A proximité du chenal de navigation et d'une écluse, il est fondamental de ne pas détériorer les conditions d'agitation ni d'accélérer la sédimentation devant l'ouvrage.

Un ouvrage de type double écran partiel semi réfléchissant est proposé pour répondre à ces contraintes.

\section{Mots-clés :}

Génie côtier, Ouvrage de protection, Extension portuaire, Paroi perforée.

\section{Introduction}

Le port de plaisance du Grand Large de Dunkerque est situé à l'intérieur de la rade de Dunkerque (voir figure 1). Son développement est contraint par la présence du chenal de navigation situé à proximité. Le contexte climatologique est tel qu'il existe une agitation importante dans le chenal et au droit du plan d'eau du port de plaisance. La protection du plan d'eau est à l'heure actuelle assurée par des brise-lames flottants positionnés à l'extrémité Est du bassin.

Afin de développer son activité, le SMDN souhaite agrandir le port de plaisance grâce à la construction d'un ouvrage de protection contre la houle en amont du chenal.

Cette extension, en lieu et place des brise-clapots actuels nécessite la réalisation d'un nouvel ouvrage de protection.

Les contraintes principales sont:

- Les contraintes liées à la compatibilité de l'opération avec la navigation ;

- Les contraintes physiques liées aux houles du large et à la propagation de clapots dans le chenal, mais aussi au marnage considérable ;

- Les contraintes liées à l'exploitation du port: entretien des quais, travaux de dragage, ... 


\section{Thème 4 - Ouvrages portuaires, offshore et de plaisance}

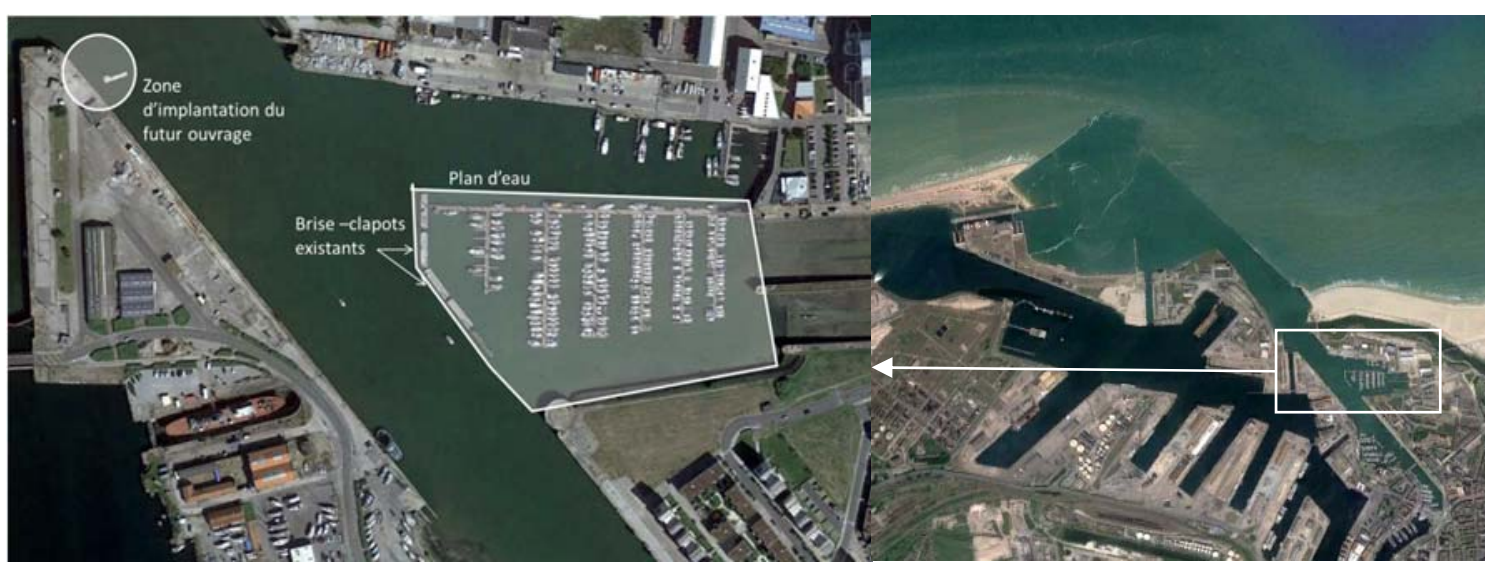

Figure 1. Contexte : port de plaisance du Grand Large de Dunkerque.

\section{Conception}

\subsection{Définition des contraintes physiques}

Des études spécifiques d'agitation ont été réalisées pour permettre de déterminer précisément les contraintes impactant le site d'étude. Le site est particulièrement soumis à des périodes pic comprises entre 3 et $6 \mathrm{~s}$ (BRL \& BOUGIS, 2012). L'ouvrage est positionné sur la rive opposée au plan d'eau, secteur de convergence des houles et clapots, de manière à atténuer ces ondes avant qu'elles ne se réfléchissent sur le quai des Monitors et impactent directement le plan d'eau.

\subsection{Optimisation de la solution}

Contraints par les activités, l'emprise et la position de l'ouvrage sont fixées. Il s'agira d'un ouvrage réalisé perpendiculairement au quai des Monitors et au chenal de navigation. L'enjeu est de proposer un ouvrage qui offrira la meilleure protection possible considérant l'agitation du secteur, le marnage et le fait qu'un prérequis est une transparence vis-à-vis du transport sédimentaire. En effet, le secteur est soumis à envasement. Aussi, il existe à proximité immédiate de l'ouvrage une écluse qu'il convient de ne pas engraisser. Enfin, la proximité avec l'écluse et le chenal de navigation impose de maintenir dans le chenal des conditions d'agitation compatibles avec ces usages.

C'est pourquoi un ouvrage semi-réfléchissant et transmissible est proposé. Il s'agit d'un ouvrage sur pieu constitué d'un écran plein partiel côté port et d'un écran perforé côté large. Tout l'enjeu consiste à définir la pénétration de l'ouvrage, la porosité de la façade avant et la largeur de l'ouvrage optimales permettant une atténuation maximale dans la chambre de dissipation ainsi crée.

Il existe un grand nombre de références bibliographiques étudiant l'efficacité hydraulique des ouvrages (BERGMANN \& OUMERACI, 1999 ; SUH et al., 2001). L'étude de TEH (2013) montre que ce type d'ouvrages est particulièrement performant 


\section{XVèmes Journées Nationales Génie Côtier - Génie Civil \\ La Rochelle, 29 au 31 mai 2018}

par rapport à d'autres solutions plus impactantes comme les caissons, de différents profils types et porosités. On peut citer parmi les articles étudiant le type d'ouvrages que l'on souhaite mettre en place l'étude de PRABISHA (2000), celle de ISAACSON et al., (1999) et celle de KOUTANDOS (2010).Une analyse bibliographique a été réalisée pour confronter ces résultats et tenter d'estimer les coefficients de transmission et de réflexion de l'ouvrage.

La transmission diminue avec la diminution de porosité et le ratio entre la profondeur d'eau et la longueur d'onde. Dans notre cas, ce rapport varie entre 0.15 et 0.7 .

De manière à protéger d'un maximum de conditions, le rapport entre la largeur de l'ouvrage et la longueur d'onde est pris entre 0.1 et 0.35 . La pénétration de l'ouvrage a été fixée pour minimiser l'impact de la circulation sur le fond. Et la porosité est fixée à $20 \%$.

L'ouvrage tel que conçu permettrait de réduire la transmission en particulier à basse mer, ne dépassant pas $20 \%$.

Considérant les résultats publiés par PRABISHA (2000), on peut estimer la réflexion associée à l'ouvrage projeté à 20 à $40 \%$ pour les conditions d'ondes courtes et jusqu'à $50 \%$ pour les conditions où la période pic s'approche de $6 \mathrm{~s}$ en pleine mer.

\subsection{Réalisation}

Pour une répartition optimale des efforts, le parti est pris de réaliser des pièces venant s'imbriquer sur les pieux 2 à 2. Rappelons qu'il existe une problématique de coûts d'ouvrage et de mise en œuvre. Il y a donc nécessité à maximiser les temps de travaux à terre. Cet ouvrage permet la préfabrication des pièces en amont du chantier pour un empilement après installation des pieux, figure 2. De manière à minimiser le nombre d'éléments différents à réaliser, un assemblage est proposé sur la base de 5 pièces. La façade avant est composée d'une combinaison d'assemblage d'une unique pièce positionnée en quinconce, figure 3.

\section{Conclusions}

L'ouvrage permet théoriquement une atténuation de l'agitation se propageant dans le chenal vers le plan d'eau tout en maintenant des conditions d'agitation dans le chenal acceptables. La structure de l'ouvrage est adaptée pour une mise en œuvre aisée. Les efforts sont répartis sur l'ouvrage pour meilleure pérennité.

Des essais physiques permettraient de valider l'efficacité hydraulique et d'optimiser le dispositif. 
Thème 4 - Ouvrages portuaires, offshore et de plaisance

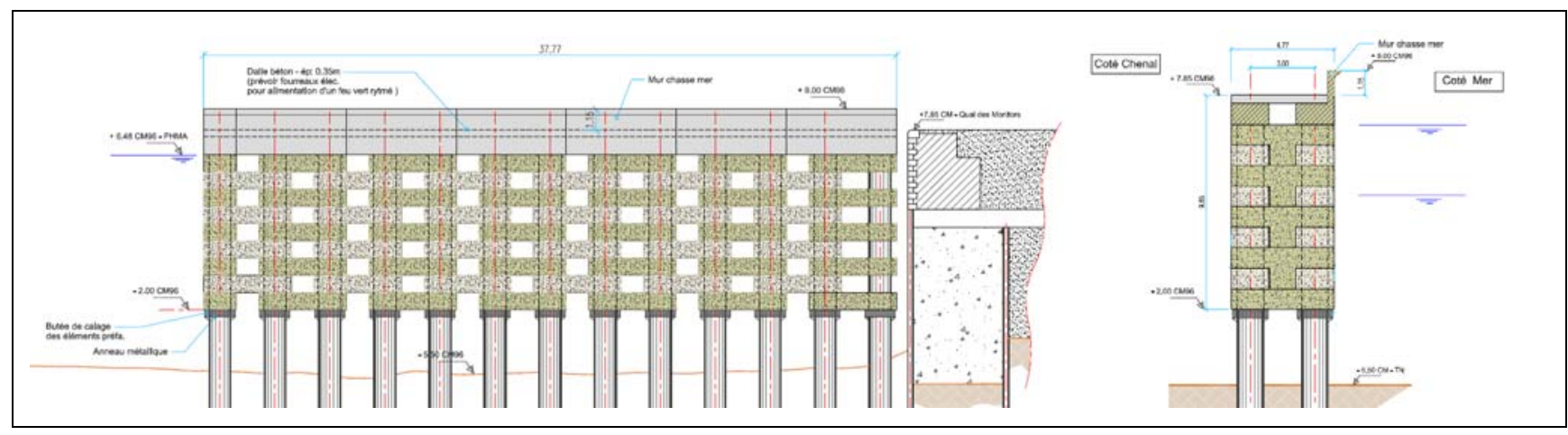

Figure 2. Ouvrage. Vue de la façade avant (à gauche). Coupe type (à droite).

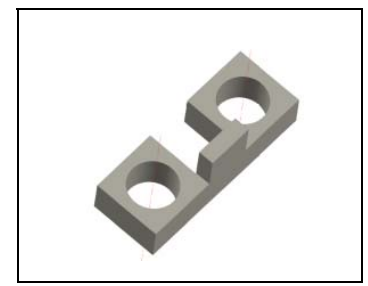

Figure 3. Exemple de vue 3D d'une pièce béton formant la façade avant de l'ouvrage.

\section{Remerciements}

L'équipe remercie Mme Barez et M. Bénard, agissant en tant que Maître d'Ouvrage respectivement pour le Port de Plaisance du Grand Large et la Communauté Urbaine de Dunkerque.

\section{Références bibliographiques}

BERGMANN H., OUMERACI H. (1999). Hydraulic performance of perforated structures. Proc. of the $5^{\text {th }}$ COPEDEC Conf., Cap Town (South Africa), $10 \mathrm{p}$.

ISAACSON M., BALDWIN J., PREMASIRI S., YANG G. (1999). Wave interaction with double slotted barriers. Applied Ocean Research, Vol. 21(2), pp 81-91. https://doi.org/10.1016/S0141-1187(98)00039-X

KOUTANDOS E.V. (2010). Hydraulic performance of double slotted barriers under regular wave attack. WSEAS Transactions on Fluid Mechanics, Vol. 5(3), pp 91-103.

PRABISHA P.R. (2000). Roll $n^{\circ}$ 091244. Department of civil engineering of Trivandum, College of Engineering, India.

SUH K.D., CHOI J.C., KIM B.H., LEE K.S. (2001). Reflection of irregular waves from perforated-wall caisson breakwaters. Coastal Engineering, Vol. 44(2). pp 141-151. https://doi.org/10.1016/S0378-3839(01)00028-X

TEH H. M. (2013). Hydraulic performance of free surface breakwaters: A review. Sains Malaysiana, Vol. 42(9), pp 1301-1310. 